\title{
Combined sorption/transport of sodium dodecyl sulfate and hydrochloric acid in a blend of cellulose acetate butyrate with cellulose acetate hydrogen phthalate
}

\author{
Artur J.M. Valente ${ }^{\text {a,* }}$, Hugh D. Burrows ${ }^{\text {a }}$, Alexandre Ya. Polishchuk ${ }^{\text {b, }}$ \\ Maria G. Miguel ${ }^{\mathrm{a}}$, Victor M.M. Lobo ${ }^{\mathrm{a}}$ \\ a Department of Chemistry, University of Coimbra, 3004-535 Coimbra, Portugal \\ b Kompamid LLC, 6 Proezd Zavoda "Serp\&Molot", Moscow 111250, Russia
}

Received 17 June 2003; received in revised form 2 September 2003; accepted 2 September 2003

\begin{abstract}
The transport of hydrochloric acid $(0.001-0.1 \mathrm{M})$ and sodium dodecyl sulfate $(0.001-0.1 \mathrm{M})$ has been measured through a membrane consisting of a blend of cellulose acetate butyrate and cellulose acetate hydrogen phthalate. The cellulose derivative blend is suggested to suffer an alteration in the degree of hydrophobicity when in equilibrium with sodium dodecyl sulfate (SDS) through hemimicelle formation. An increase in surface hydrophobicity of the blend when in equilibrium with SDS solution was observed by fluorescence measurements using the vibronic bands of the probe pyrene, as well as by water desorption kinetics; a decrease of the effective diffusion coefficients from $1.2 \times 10^{-11} \mathrm{~m}^{2} \mathrm{~s}^{-1}$ in the absence of SDS to approximately $2 \times 10^{-13} \mathrm{~m}^{2} \mathrm{~s}^{-1}$ in its presence was found. The value obtained for the mutual diffusion coefficient of $\mathrm{HCl}$ in the concentration range 0.001-0.1 $\mathrm{M}\left(D=4.2 \times 10^{-14} \mathrm{~m}^{2} \mathrm{~s}^{-1}\right)$ shows also that the membrane presents hydrophobic features. The flux of SDS in the blend membrane at different $\mathrm{pH}$ values shows two distinct permeation rates depending on the cmc. However, from the calculation of permeability coefficients at SDS concentrations below the $\mathrm{cmc}$ a clear decrease in $\mathrm{P}$ is found, whilst, at concentrations above the cmc the permeability coefficients are nearly constant, only showing a slightly increase. The diffusion coefficients of SDS in the blend increase over the whole SDS concentration range analysed and show an effective diffusion coefficient 2-3 orders of magnitude below the diffusion coefficients of SDS in aqueous solutions. This fact suggests that the only diffusing species are SDS unimers. The presence of $\mathrm{HCl}$ in the SDS bulk solution has the effect of increasing the permeability and diffusion coefficients. Mutual analysis of permeation and diffusion coefficients and sorption isotherms shows that, on decreasing the $\mathrm{pH}$, the interactions between SDS and the polymer network decrease. This is also reflected in a clear decrease of the hydrophobic interactions between the diffusing and polymeric species, provoked by a decrease in the unimer-unimer association.
\end{abstract}

(c) 2003 Elsevier Ltd. All rights reserved.

Keywords: Diffusion coefficients; Sodium dodecyl sulfate; Cellulose esters; Surfactants

\footnotetext{
${ }^{*}$ Corresponding author. Tel.: +351-239-8852080; fax: +351239-827703.

E-mail address: avalente@ci.uc.pt (A.J.M. Valente).
}

\section{Introduction}

Polymer membranes find applications in areas as varied as separation science [1], sensors [2] and surface coatings [3]. Particular interest is devoted to surfactantpolymer systems relevant to various areas, including formation of gels for use as thickeners [4-8], and in 
textile [9] and paper [10] processing. Diffusion of electrolytes across such membranes is important in many of those areas, such as in optimising conditions for separation processes and understanding the basic mechanisms involved.

We have previously reported [11] that cellulose ester blends, based on cellulose acetate butyrate (CAB) and cellulose acetate hydrogen phthalate (CAHP), show an interesting selectivity to SDS permeation, which depends on the hydrophilic/hydrophobic balance in the blend composition and on the temperature. In order to study the effect of SDS micellization as well as to extend the analysed SDS concentration range to more dilute concentrations, we have chosen the more hydrophilic blend; the effect of acidification of the bulk solution is also studied to check its effect in SDS structure and in the polymeric blend. Our final scope is to find appropriate membranes for separation by either changing the hydrophobic/hydrophilic balance in the blend, or altering in a simple way (e.g., changing $\mathrm{pH}$ ) the solution.

The transport of SDS is analysed by a method originally developed for calculating diffusion coefficients of electrolytes [12-14] from electrochemical conductivity measurements. This method is applied to the study of diffusion of electrolytes in polymer membranes $[13,14]$. We are particularly interested in the behaviour of ionic surfactants, and we have previously studied the diffusion of the important anionic detergent sodium dodecyl sulfate (SDS) in poly(acrylamide) gels [15] and in cellulose ester membranes of differing degrees of hydrophobicity [11].

We will show how $\mathrm{pH}$ alterations might affect the SDS permeation. Experiments were carried out using solutions of $\operatorname{SDS}\left(10^{-3}-10^{-1} \mathrm{M}\right)$ mixed with $\mathrm{HCl}\left(10^{-3}\right.$ $\left.10^{-1} \mathrm{M}\right)$ to evaluate how the $\mathrm{pH}$ affects the SDS permeation. In addition to their application to the present system, these studies are also important in more general terms since the transport of mixed solutes has received little attention and is a very important feature for the characterisation of mass transport processes occurring in polymeric matrices.

\section{Experimental}

\subsection{Reagents}

The chemicals used were from the following sources: cellulose acetate butyrate $(\mathrm{CAB})$ containing $17 \%$ butyrate, cellulose acetate hydrogen phthalate (CAHP) and tetrahydrofuran $+99.9 \%$ (THF) from Aldrich; sodium dodecyl sulfate (SDS) from Merck; and potassium chloride and hydrochloric acid 32\% from Riedel deHäen. All low-molecular weight compounds are of ProAnalysis quality and were used without further pu- rification. The solid $\mathrm{KCl}$ was weighed after drying the salt until constant weight at $110{ }^{\circ} \mathrm{C}$.

Concentrated SDS solution was obtained by dissolving the corresponding amount of solid in bi-distilled water of conductivity $1.2(0.4) \times 10^{-4} \Omega^{-1} \mathrm{~m}^{-1}$, and standard solutions of different concentrations were prepared from this by dilution.

\subsection{Membrane preparation}

The CAB/CAHP blend films were obtained by initially dissolving CAB $(33.3 \% \mathrm{w} / \mathrm{v})$ and CAHP $(66.7 \%$ $\mathrm{w} / \mathrm{v}$ ) in THF, at a concentration of $10 \%$ (weight of polymer/volume of solvent) and stirring for $24 \mathrm{~h}$ to ensure homogeneity. The homogeneous solution was then deposited as a film on a flat glass support using a Simex automatic film applicator. Specific moulds were used to prepare membranes with a homogeneous thickness. After complete evaporation of the solvent at room temperature, the membrane was removed from the glass support with the help of water. Characterisation of the blend shows a certain degree of mixing of the two polymers, since the density of the blend $\left(0.850 \mathrm{~g} \mathrm{~cm}^{-3}\right)$ is lower than that of either CAHP $\left(0.902 \mathrm{~g} \mathrm{~cm}^{-3}\right)$ or CAB $\left(0.940 \mathrm{~g} \mathrm{~cm}^{-3}\right)$, and the differential scanning calorimetry (DSC) curve of the blend film is also different from that of the two pure polymers (Fig. 1).

\subsection{Differential scanning calorimetric analysis}

The samples ( $2 \mathrm{mg}$ weight) were cut from films which were prepared as described above. To remove the residual solvent, the films of pure polymers and blend were dried at reduced pressure for, at least, one day at ambient temperature. Differential scanning calorimetry measurements were performed using a Shimadzu differential scanning calorimeter, $D S C-50$ model, at a heating

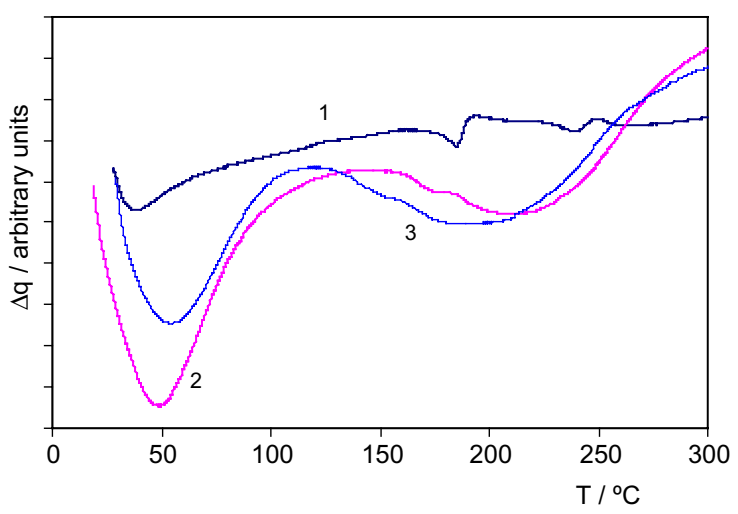

Fig. 1. DSC curves obtained for films of cellulose acetate butyrate (1), cellulose acetate hydrogen phthalate (2) and CAB/ CAHP blend (3). 
rate of $20^{\circ} \mathrm{C} / \mathrm{min}$, in a nitrogen atmosphere flowing at $20 \mathrm{~mL} / \mathrm{min}$. The thermograms (Fig. 1) were obtained after the following treatment: heating from ambient temperature to $300{ }^{\circ} \mathrm{C}$ and posterior cooling to ambient temperature and maintained on this temperature for $5 \mathrm{~min}$.

\subsection{Permeation experiments}

The transport of SDS, $\mathrm{HCl}$ and their mixture through the membranes was measured by using a permeation cell as described previously [16,17]. This consists of two cells filled with a permeant solution (cell A) and water (cell B). These are connected by two horizontal tubes, and the polymer membrane is placed between them. Silicone was used to seal the membrane to ensure hermetic interfaces. The experiments were carried out ensuring that there is streamlined flow near the membrane, and that there is no hydrostatic pressure influencing the transport.

The variation of concentrations of binary systems of SDS and $\mathrm{HCl}$ was determined in cell $\mathrm{B}$ during permeability experiments by measuring the electrical conductivity using a YSI 3200 instrument. During the multicomponent transport of $\mathrm{SDS} / \mathrm{HCl}$ mixtures, the flux of the diffusing species was monitored both by measuring the electrical conductivity and by a potentiometric technique using a liquid membrane chloride selective (Orion, Ref. 93-17 with a detection limit of $\left.5 \times 10^{-6} \mathrm{M}\right)$ and combined $\mathrm{pH}$ electrodes, with a Radiometer PHM240 pH meter ( $\mathrm{pH}$ resolution 0.001 , electrode potential resolution $0.1 \mathrm{mV}$ ). All the $\mathrm{pH}$ values were corrected by measuring the electrode sensitivity as well as the zero $\mathrm{pH}$. The variation of electrical conductivity in cell B was monitored continuously. However, it was not possible to use this approach for $\mathrm{pH}$ and chloride electrode potential measurements because of possible artefacts caused by doping of the electrodes at the electrolyte concentrations studied over the long time scales used in these studies. Instead, the following procedure was used: after the beginning of each experiment, $10 \mathrm{~mL}$ aliquots of solution were taken from cell $\mathrm{B}$ at fixed intervals of time. These were diluted with bi-distilled water and the chloride and hydrogen ion concentrations were measured potentiometrically. The total ion concentration in cell $\mathrm{B}$ was calculated from the electrical conductivity data, while the concentrations of $\mathrm{H}^{+}$and $\mathrm{Cl}^{-}$were obtained from the potentiometric measurements. Within experimental error, the proton and chloride concentrations were identical, showing that $\mathrm{HCl}$ can be considered to diffuse as a single entity. Thus, according the electroneutrality principle [18], the SDS concentration can be calculated subtracting the concentration of $\mathrm{HCl}$ from the total concentration of diffusing species.

The flux of each component of the solution, $J_{i}$, with $i$ representing $\mathrm{HCl}$ or SDS, was calculated according to
$J_{i}=(V / A)\left(\mathrm{d} c_{i} / \mathrm{d} t\right)$

where $V$ and $A$ are the volume of the solution in cell B $(200 \mathrm{~mL})$ and the permeating area $\left(1.54 \mathrm{~cm}^{2}\right)$, and $\left(\mathrm{d} c_{i} / \mathrm{d} t\right)$ is the variation of the concentration of the $i$-species, in cell $\mathrm{B}$, with time $t$. The thickness of each membrane $(l \cong 20 \mu \mathrm{m})$ was measured after each experiment and showed no variation resulting from swelling.

The conductivity and potentiometry instruments were calibrated (i.e., the dependence of the electrical conductivity, $\mathrm{pH}$ and electrode potential on the SDS, $\mathrm{HCl}$ and $\mathrm{KCl}$ concentrations, respectively) prior to each experiment using, at least, four standard solutions, whose concentration range covered the range of experimental values being measured in cell $\mathrm{B}$. The concentrations of $\mathrm{HCl}$ solutions were accurately determined by volumetric titration with a standard solution of sodium tetraborate.

The same experimental conditions were used for calibration and permeability experiments: solutions were stirred at $\approx 220 \mathrm{rpm}$, and constant temperature $(25 \pm 0.1$ ${ }^{\circ} \mathrm{C}$ ) was maintained by using a VelpScientifica Multistirrer 6 thermostat bath. Data were recorded over a sufficient time to ensure a steady-state flux, but such that the diffusant concentration in cell $\mathrm{B}$ was always well below the cell A concentration $(<10 \%)$.

\subsection{Water desorption}

All gravimetric measurements on water desorption were made to $\pm 0.1 \mathrm{mg}$ using a Sartorius Analytical balance. Different samples of the same membrane were placed in water for approximately 2 weeks. Following this, the membranes were rinsed with distilled water, excess moisture was wiped off, and samples placed inside a $10^{-2}$ M SDS solution for $120 \mathrm{~h}$. After this equilibrium time (gravimetrically controlled) the membranes were removed and excess moisture was wiped off. The loss of weight with time under vacuum at $25^{\circ} \mathrm{C}$ was monitored gravimetrically. The weight/weight $(\mathrm{w} / \mathrm{w})$ water concentration in the membrane, $C_{\mathrm{w} t}$, at time, $t$, was calculated from

$C_{\mathrm{w} t}=M_{t}-M_{\infty} / M_{t}$

where $M_{t}$ is the weight of the sample at time $t$ and $M_{\infty}$ is the weight of the dried sample, obtained after the complete desorption of water to a constant value. The initial weight/weight $(\mathrm{w} / \mathrm{w})$ water concentration $\left(C_{\mathrm{w} 0}\right)$ is obtained at $t=0$.

The effective water diffusion coefficients, $D_{\text {weff }}$, were computed using a Fickian approach to fitting the water desorption curve (Fig. 2)

$1-\left(C_{\mathrm{w} t} / C_{\mathrm{w} 0}\right)=4\left(D_{\mathrm{weff}} t / \pi l^{2}\right)^{0.5}$ 


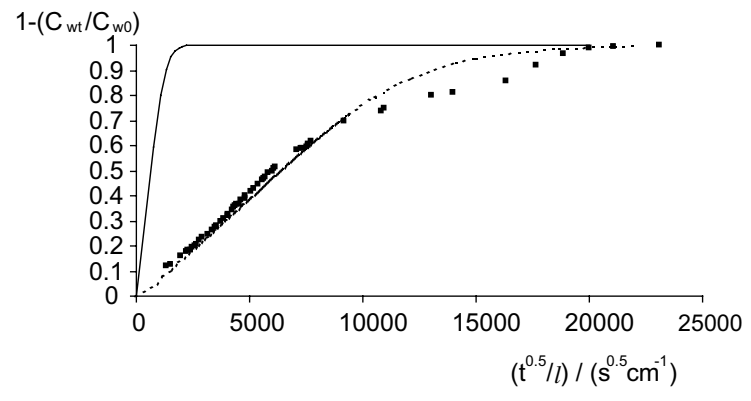

Fig. 2. Desorption kinetics of water from $\mathrm{CAB} / \mathrm{CAHP}$ blend membranes in water (-), [11] and in $10^{-2} \mathrm{M}$ SDS solution (๑). The dashed lines were obtained from fitting Eq. (3) to the experimental data.

where $l(=0.040 \mathrm{~mm})$ is the thickness of the membrane, measured using a Helias micrometer $(0.005 \mathrm{~mm})$.

\subsection{Sorption experiments}

Different samples (square geometry of well-defined area, $A^{\prime}=25 \mathrm{~cm}^{2}$ ) of the same membranes were cut, immersed in water at $25{ }^{\circ} \mathrm{C}$ until equilibrium was reached ( 2 weeks). Following this, they were transferred to SDS, $\mathrm{HCl}$ or mixture solutions, with volumes of 40 $\mathrm{mL}$, and left to reach equilibrium (3 weeks). Subsequently, the membranes were removed and their thickness, $l$, was measured. When no more swelling was observed, the polymer volume, $V_{\mathrm{P}}$, was then calculated from $A^{\prime}$ and $l$. The approach to equilibrium was controlled gravimetrically.

The concentration of the SDS, $\mathrm{HCl}$ or $\mathrm{SDS}+\mathrm{HCl}$ sorbed by the membrane, $C$, was calculated using

$C=\left(c_{\infty}-c_{0}\right) V^{\prime} / V_{\mathrm{P}}$

by measuring the concentration of the sorbed species in aqueous solution of a volume $V^{\prime}$ prior $\left(c_{0}\right)$ and after $\left(c_{\infty}\right)$ the sorption experiments, using the same techniques described in the previous section.

The average values of $C$ were obtained from three independent measurements.

\subsection{Fluorescence measurements}

Fluorescence measurements on membranes were made using a Spex Fluorolog 111 spectrometer, with samples mounted in $1 \mathrm{~cm}^{2}$ quartz cuvettes and excitation at $337 \mathrm{~nm}$. A sample of the polymeric blend was immersed in an aqueous solution of pyrene (Py, $\left.10^{-5} \mathrm{M}\right)$ in SDS $(10 \mathrm{mM})$ overnight, removed and then its fluorescence spectrum measured. Fluorescence was also used to study diffusion of SDS across the membranes by measuring the spectra of aliquots of solution from cell B in $1 \mathrm{~cm}^{2}$ quartz cuvettes.

\section{Results and discussion}

\subsection{Effect of SDS on polymer blend hydrophobicity}

Fluorescence studies were carried out on the membranes using pyrene (Py) as a probe, and studying the spectrum after immersion of the membrane in SDS solution. Relative intensities, $I_{1} / I_{3}$, of the first and third vibronic bands of pyrene fluorescence, which are a measure of local polarity $[19,20]$, were measured for the systems Py/polymer membrane/SDS as a function of immersion times of the membrane samples in SDS (0.01 M) solution. When the immersion times of the membrane in SDS solution were 1, 7 and 11 days the corresponding relative intensities $I_{1} / I_{3}$ were $1.59( \pm 0.02), 1.43$ $( \pm 0.01)$ and $1.32( \pm 0.02)$, respectively. These results show that there is an increase of the surface hydrophobicity of the polymer [19] with time of immersion of the membrane. The effect of the surfactant on the membrane hydrophobicity can be accounted for using the concept of hemimicelle (HM) formation [21]. Hemimicelles have previously been observed on adsorption of SDS on solid surfaces, such as alumina, by fluorescence probes [22], electron spin resonance spectrocopy using nitroxide spin probes [23] and excited-state resonance Raman spectroscopy [24]. Once the negatively-charged head groups of the surfactant molecules show the same charge than those of carboxylic group of CAHP, it is expected that the hydrocarbon chains of the surfactant ions can be adsorbed by the hydrophobic structure of $\mathrm{CAB}$ in order to reduce the free energy of the system, and consequently a HM formation occurs (Fig. 3a).

Having shown that surfactant adsorption increases hydrophobicity of the membranes, the following step was to evaluate how this can affect the blend transport properties, especially in terms of the permeation features.

Support for membrane modification comes from studies of the kinetics of water desorption from the blend (Fig. 2). Both, the rate and mechanism of water desorption show differences in the presence and absence of SDS, showing that the SDS $(10 \mathrm{mM})$ alters the membrane. The effective water diffusion coefficient, $D_{\text {weff }}$, for short-range times [25], as obtained by desorption measurements, decreases from $1.2( \pm 0.2) \times 10^{-11}$ $\mathrm{m}^{2} \mathrm{~s}^{-1}$ (without SDS) to $1.3( \pm 0.2) \times 10^{-13} \mathrm{~m}^{2} \mathrm{~s}^{-1}$, in the presence of SDS solution. The water concentration in blend $C_{\mathrm{w}}$ decreases from approximately $20 \%(\mathrm{w} / \mathrm{w})$ to

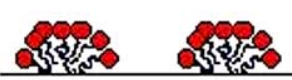

(a)

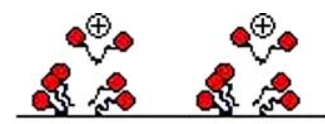

(b)
Fig. 3. Schematic representation of hemimicelle formation on the blend surface without (a) and with (b) $\mathrm{HCl}$. 
approximately $16 \%(\mathrm{w} / \mathrm{w})$ when in contact with SDS solution as a direct consequence of the increase in hydrophobicity. The shape of such a kinetic plot also shows at $1-C_{\mathrm{w} t} / C_{\mathrm{w} \infty}>0.6$ a deviation from Fickian behaviour (dashed line in Fig. 2). A possible explanation for such non-Fickian behaviour, with a negative deviation from the theoretical curve, can be due to the occurrence of relaxation phenomena [26] of the polymeric matrix during water desorption. This hypothesis is based on experimental findings of rehological studies of surfactant-urethane ethoxylated hydrophobically modified systems [27].

Since the proposed interaction mechanism of HM formation between SDS and the polymer suggests that an additional resistance to the SDS permeation will occur [28], an estimation of the thickness of such layer was attempted. The analysis of the Fig. 4 shows an unusual increase of SDS flux $\left(J_{\text {SDS }}\right)$ with an increase of the membrane thickness $(l)$. This behaviour shows that blend features, such as the polar groups of the CAHP as well as the amphiphilic properties of the cellulose structure $[13,29,30]$, are also very important and cannot be neglected in the overall diffusion process analysis. For example, although the cellulose ester membrane shows

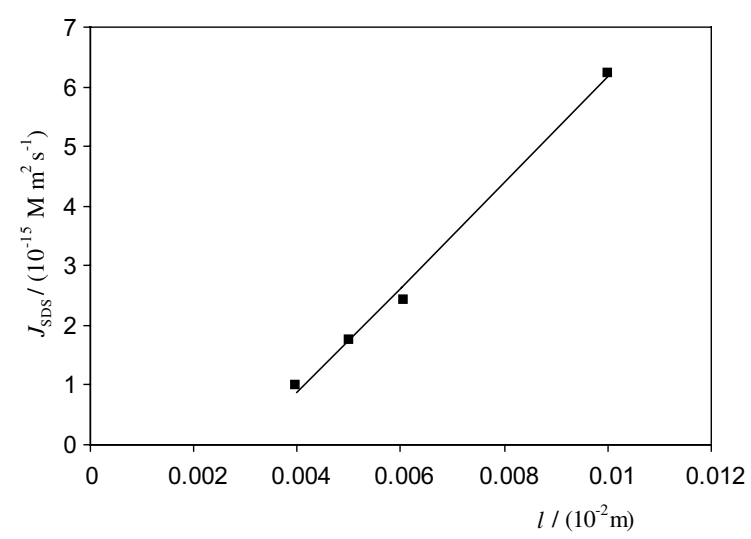

Fig. 4. Effect of membrane thickness, $l$, of CAB/CAHP blend membrane on the flux, $J_{\mathrm{SDS}}$ of SDS $10 \mathrm{mM}$ in the first 2 days of experiments in steady-state conditions. zones with different degrees of crystallinity, the surface presents pores and rough zones, and the bulk of the film does not show any large pores [31], the spongy structure of cellulose derivatives [32] might play an important role in diffusion processes.

A critical discussion of the balance effect between the different properties mentioned on the permeation of SDS and/or $\mathrm{HCl}$ will be presented in the next sections.

\subsection{Sorption isotherms of $S D S$}

The equilibrium sorption concentrations of SDS, $C_{\mathrm{SDS}}$, in $\mathrm{CAB} / \mathrm{CAHP}$ membranes at different $\mathrm{pH}$ conditions are shown in Table 1. The concentration of the SDS increases with the equilibrium $\mathrm{HCl}$ concentration in the system, showing that the sorption of the SDS unimer form increases as a consequence of a decrease in the SDS association, which can be provoked either by the presence of hydrogen ions or of the sulfate groups of SDS hemimicelles (Fig. 3). In the absence of $\mathrm{HCl}$, only a slight increase in $C_{\mathrm{SDS}}$ is found with $c$, noticeably at concentrations near or above the critical micelle concentration $\left(\mathrm{cmc}=8.16 \times 10^{-3} \mathrm{M}\right.$ [33]). The sorption of SDS can occur by two different mechanisms via unimer and/or by interaction between the hydrophobic part of blend and the SDS with consequent hemimicelle formation. The latter mechanism is supported by the high distribution coefficients, observed particularly at SDS concentrations below $1 \times 10^{-2} \mathrm{M}$. The sorption (by partition) of unimer by the polymeric matrix results from the fact that SDS can permeate the blend membrane only in the unimer form. Confirmation of this has come from studies of permeation across polymer membranes carried out using the fluorescence of pyrene incorporated in aqueous SDS solutions at concentrations above the cmc. After $168 \mathrm{~h}$ following the beginning of the permeation experiment there are no indications of pyrene fluorescence in the solution in water cell, confirming that micelles do not permeate the polymer membrane. As a consequence, the results in Table 1 suggest that micelles may act by influencing the entrance of the unimers into membrane.

Table 1

Equilibrium sorption of SDS aqueous solutions by the CAB/CAHP blend at different $\mathrm{HCl}$ concentrations, at $25^{\circ} \mathrm{C}$

\begin{tabular}{lllll}
\hline$c_{\mathrm{SDS}} / \mathrm{M}$ & \multicolumn{3}{l}{$C_{\mathrm{SDS}} / \mathrm{M}$} & \\
\cline { 2 - 5 } & $c_{\mathrm{HCl}}(s)=0 \mathrm{M}$ & $c_{\mathrm{HCl}}(s)=0.001 \mathrm{M}$ & $c_{\mathrm{HCl}}(s)=0.01 \mathrm{M}$ & $c_{\mathrm{HCl}}(s)=0.1 \mathrm{M}$ \\
\hline 0.001 & $0.025(0.002)$ & $0.071(0.002)$ & $0.128(0.003)$ & $0.208(0.003)$ \\
0.005 & $0.025(0.002)$ & $0.086(0.003)$ & $0.187(0.002)$ & $0.235(0.004)$ \\
0.01 & $0.026(0.002)$ & $0.091(0.005)$ & $0.211(0.005)$ & $0.255(0.005)$ \\
0.05 & $0.038(0.002)$ & $0.135(0.006)$ & $0.316(0.003)$ & $0.375(0.002)$ \\
0.1 & $0.051(0.003)$ & $0.181(0.006)$ & $0.432(0.003)$ & $0.535(0.004)$ \\
\hline
\end{tabular}

$s$-standard deviation of, at least, three independent measurements. 
A dual mode isotherm is found to reasonably explain the SDS sorption: in a presence of $\mathrm{HCl}$ the SDS concentration tends towards the limit of Henry's law, showing that the permeation of SDS is clearly dependent both on solubility and on the mobile fraction of the sorbed molecules (Eq. (5))

$C_{\mathrm{SDS}}=K^{\prime} c_{\mathrm{SDS}}+K_{0}^{\prime}$

where $K^{\prime}$ and $K_{0}^{\prime}$ are constants related with the Henry's law dissolution and site saturation concentration, respectively. If we assume the sorption isotherms of SDS are part of a dual mode sorption then $K_{0}^{\prime}=C_{\infty}$. The values of $K^{\prime}$, obtained from fitting the experimental data (Table 1) to Eq. (5), are the following: $K^{\prime}(\mathrm{HCl}, 0 \mathrm{M})=$ $0.27( \pm 0.01), K^{\prime}(\mathrm{HCl}, 0.001 \mathrm{M})=1.06( \pm 0.07), K^{\prime}(\mathrm{HCl}$, $0.01 \mathrm{M})=2.79( \pm 0.32)$ and $K^{\prime}(\mathrm{HCl}, 0.1 \mathrm{M})=3.15( \pm 0.43)$. These results show that the SDS dissolution inside polymeric matrix increases when $\mathrm{HCl}$ concentration also increases.

\subsection{Sorption isotherms of $\mathrm{HCl}$}

The results of $\mathrm{HCl}$ sorption by the blend material (Fig. 5), either from aqueous solution or from $\mathrm{HCl}-\mathrm{SDS}$ mixture, can be reasonably treated in terms of a Langmuir type isotherm (Eq. (6)),

$C_{\mathrm{im}}=K_{1} C_{\infty} C_{\mathrm{f}} /\left(1+K_{1} C_{\mathrm{f}}\right)$

where $C_{\mathrm{im}}$ is the concentration of the immobilised sorbed molecules, $C_{\infty}$ is the concentration of the sorbed molecules which can interact with the polymer, and $K_{1}$ is an equilibrium constant involving the sorption and desorption processes. The concentration of free molecules inside polymer, $C_{\mathrm{f}}$, is assumed to be linked to the concentration in the bulk solution, $c$, through a Henry's law type equation

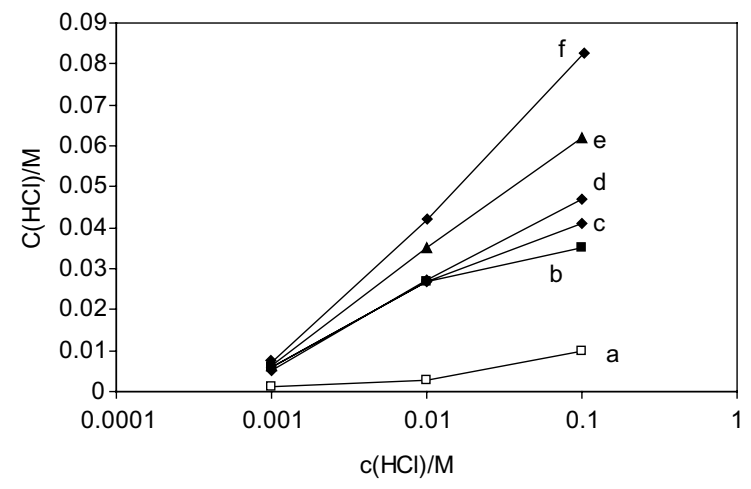

Fig. 5. Dependence of the $\mathrm{HCl}$ concentration inside polymeric membrane, $C$, on the $\mathrm{HCl}$ bulk solution concentration, $c$, in the presence of SDS at different concentrations: (a) $0 \mathrm{M}$; (b) 0.001 $\mathrm{M}$; (c) $0.005 \mathrm{M}$; (d) $0.01 \mathrm{M}$; (e) $0.05 \mathrm{M}$; (f) $0.1 \mathrm{M}$. The lines are only to guide the eyes.
Table 2

Langmuir isotherm parameters computed from fitting Eq. (8) to the experimental data shown in sorption isotherms of $\mathrm{HCl}$ (Fig. 5)

\begin{tabular}{llll}
\hline$c_{\mathrm{SDS}} / \mathrm{M}$ & $K_{2}$ & $C_{\infty} / \mathrm{M}$ & $R^{2 \mathrm{a}}$ \\
\hline 0 & 287 & 0.0053 & 1.00 \\
0.001 & 213 & 0.034 & 1.00 \\
0.005 & 190 & 0.037 & 0.99 \\
0.01 & 128 & 0.043 & 1.00 \\
0.05 & 114 & 0.063 & 1.00 \\
0.1 & 90 & 0.086 & 1.00 \\
\hline
\end{tabular}

${ }^{\mathrm{a}} R^{2}$ - correlation coefficient.

$C_{\mathrm{f}}=K_{0} c$

where $K_{0}$ is a partition coefficient. Following Eqs. (6) and (7), Eq. (8) represents the above dependence in terms of reciprocal co-ordinates,

$1 / C_{\mathrm{im}}=1 / C_{\infty}+\left[1 /\left(K_{2} C_{\infty}\right)\right](1 / c)$

where $K_{2}=K_{0} K_{1}$.

Table 2 presents the parameters of $\mathrm{HCl}$ sorption by polymer blend. The experimental data show an excellent fit to Eq. (8). From these results, and taking into account the experimental results of SDS sorption isotherms, shown in previous section, the following conclusions can be drawn:

- The sorption of $\mathrm{HCl}$ by the polymeric structure shows a very low $C_{\infty}$, indicating that only a small part of the acid interact with the carboxylic groups of the blend.

- As the SDS concentration increases, the formation of hemimicelles increases; under these circumstances the hydrogen ions will interact with the SDS-sulfate group, decreasing the SDS aggregation and increasing the hydrophilicity of polymer.

- As a consequence, the amount of SDS unimers which may go in the polymeric matrix will increase, and the unimer-unimer interactions will become stronger at higher SDS concentrations.

- An increase in the SDS concentration clearly results in a decrease in the rate constant for $\mathrm{HCl}$ sorption, supporting the assumption that SDS molecules may compete with $\mathrm{HCl}$ for interaction with polymer groups.

\subsection{Transport of hydrochloric acid}

Permeation experiments on $\mathrm{HCl}$ in the polymer membranes show that a steady-state flux is reached within the first $11 \mathrm{~h}$ of the experiment and is maintained during 2 days.

However, in the presence of aqueous solutions of SDS, we have shown that there is an alteration of the cellulose 
ester membrane structure, and we suspect that the contact between $\mathrm{HCl}$ and the polymer blend will be followed by some specific interaction between, for example, the hydrogen ions and the carboxylic groups of CAHP. Such an interaction may result either in a complete immobilization of a fraction of diffusant molecules or their participation in processes leading to their binding to certain sites in the polymeric matrix. Even in the absence of any other complicating circumstances, the effective diffusion coefficients will depend on the rate constants of these reactions.

Assuming immobilisation of free $\mathrm{HCl}$ molecules

$C=C_{\mathrm{f}}+C_{\mathrm{im}}$

where $C$ is the total concentration, $C_{\mathrm{f}}$ is the concentration of free molecules, $C_{\mathrm{im}}$ is the concentration of immobilised molecules $\left(C_{\mathrm{im}} \neq 0\right)$, we can write the flux of this species as

$J=-D \partial C_{\mathrm{f}} / \partial x=-D_{\text {eff }} \partial^{2} C / \partial x^{2}$

and deduce an effective diffusion coefficient

$D_{\text {eff }}=D\left(\partial C / \partial C_{\mathrm{f}}\right)^{-1}$

If the concentrations of mobile and immobile molecules are directly proportional $\left(C_{\mathrm{im}}=K C_{\mathrm{f}}\right)$, then the effective diffusion coefficients remain constant: $D_{\text {eff }}=[D /$ $(1+K)]$. If we assume a limited number of specific sites (as, for example, in the Langmuir mechanism of sorption), $D_{\text {eff }}$ will increase with concentration of diffusing species, with values approaching diffusion coefficient of free molecules. Using membranes of 0.040-mm thickness, the $D_{\text {eff }}$, for $\mathrm{HCl} 0.001,0.01$ and $0.1 \mathrm{M}$, are $0.71 \times 10^{-14}$, $3.0 \times 10^{-14}$ and $4.8 \times 10^{-14} \mathrm{~m}^{2} \mathrm{~s}^{-1}$, respectively.

The diffusion coefficient $D$ of $\mathrm{HCl}$ in CAB/CAHP blend at $C_{\mathrm{SDS}}=0$ can be calculated using the parameters from the Langmuir sorption calculated above and the effective diffusion coefficients $D_{\text {eff }}$. Using the derivative $\left(\partial C / \partial C_{\mathrm{f}}\right)$ from Eqs. (6) and (10), we obtain

$D=D_{\text {eff }}\left(K_{0}+K_{1} C /\left(1+K^{\prime} c\right)^{2}\right)$

The diffusion coefficient calculated using Eq. (11) is $D=4.92 \times 10^{-14} \mathrm{~m}^{2} \mathrm{~s}^{-1}$, and shows a high resistance of the blend to the transport of $\mathrm{HCl}$. From Eq. (11) we can also calculate $K_{0}(=0.15)$, showing that a small part of the sorbed molecules are free to diffuse.

\subsection{Diffusion of SDS at different $\mathrm{HCl}$ concentrations}

Analysis of SDS permeation in the $\mathrm{HCl}$ mixtures are not easy to interpret, particularly when we are working with a quaternary system. Such analysis is further complicated by other phenomena such as aggregation equilibrium between monomers and micelles, at SDS concentrations above cmc and possible chemical reactions between some of the species in solution. Taking into account such difficulties, and once the SDS flux through the polymer blend was obtained in an independent form, we have decided to describe the permeability of SDS in terms of a simple diffusion process

$\partial C / \partial t=\partial / \partial x\left(D_{\mathrm{F}} \partial C / \partial x\right)$

with the boundary and initial conditions $C(0, t)=C_{\mathrm{SDS}}$, $C(l, t)=0$, (where $C_{\mathrm{SDS}}$ is the concentration of the surfactant in the membrane) and $C(x, 0)=0$, resulting in the simple formulae for calculation of the permeability $\left(P_{\mathrm{S}}\right)$ and apparent diffusion $\left(D_{\mathrm{F}}\right)$ coefficients [11]

$P_{\mathrm{S}}=J_{\mathrm{SDS}} l / c_{\mathrm{SDS}}$

$D_{\mathrm{F}}=l^{2} / 6 \theta$

where $J_{\mathrm{SDS}}$ is the steady-state flux of SDS through the membrane, $\theta$ is its time-lag, and $c_{\mathrm{SDS}}$ is the bulk concentration of the SDS.

As pointed out earlier, fluorescence measurements in permeation experiments of SDS at concentrations above the cmc have shown that the diffusing species permeating the polymer membranes are only surfactant monomers. As a consequence, the apparent diffusion coefficient is that due to the monomer $\left(D_{\mathrm{F}}=D_{\mathrm{m}}\right)$. This also shows that, at $c_{\mathrm{SDS}}>\mathrm{cmc}$, Eq. (14) can be rewritten as

$P_{\mathrm{S}}=J_{\mathrm{SDS}} l /[\mathrm{SDS}]_{\mathrm{cmc}}$

The critical micelle concentration used in Eq. (15) is $[\mathrm{SDS}]_{\mathrm{cmc}}=8.16 \times 10^{-3} \mathrm{M}$ [33]. Table 3 shows the flux $\left(J_{\mathrm{SDS}}\right)$, permeability $\left(P_{\mathrm{S}}\right)$ and apparent diffusion $\left(D_{\mathrm{m}}\right)$ coefficients of SDS through a CAB/CAHP blend, at different $\mathrm{HCl}$ concentrations.

The flux of SDS through polymeric blend increases with SDS concentration showing that the SDS concentration inside polymer is an important factor in this process. We can also observe, from Table 2, that $J_{\text {SDS }}$ clearly increase when the $\mathrm{HCl}$ concentration also increase (for example, $J_{\mathrm{SDS}=0.1 \mathrm{M}}(\mathrm{HCl}=0 \mathrm{M})=0.82 \times 10^{-7}$ $\mathrm{mol} \mathrm{m}{ }^{-2} \mathrm{~s}^{-1}$ and $J_{\mathrm{SDS}=0.1 \mathrm{M}}(\mathrm{HCl}=0.1 \mathrm{M})=4.61 \times 10^{-7}$ mol $\mathrm{m}^{-2} \mathrm{~s}^{-1}$ ), showing that the SDS amount free to move inside the blend matrix also increased. It also possible to conclude that the $J_{\mathrm{SDS}}$ show two distinct rates of permeation $\left(\mathrm{d} J_{\mathrm{SDS}} / \mathrm{d} c_{\mathrm{SDS}}\right)$ : one relatively high at the low SDS concentrations (for example, at $c_{\mathrm{HCl}}=0$, $\mathrm{d} J_{\text {SDS }} / \mathrm{d} c_{\text {SDS }}=11.07 \times 10^{-10} \mathrm{~m} \mathrm{~s}^{-1}$ ) and the other relatively low at SDS concentrations higher than 0.01 $\mathrm{M}$ (for example, at $c_{\mathrm{HCl}}=0, \mathrm{~d} J_{\mathrm{SDS}} / \mathrm{d} c_{\mathrm{SDS}}=2.402 \times 10^{-10} \mathrm{~m} \mathrm{~s}^{-1}$ ). The intersecting point of these two distinct rates is approximately $0.010 \mathrm{M}$ (very close to the SDS $\mathrm{cmc}$ ), showing that the faster flux is due to the unimers, and the slower flux is due to the presence of micelles which can also favour new kinds of interactions, particularly those between these new entities and the non-polar structure of the cellulose-based blend [34]. 


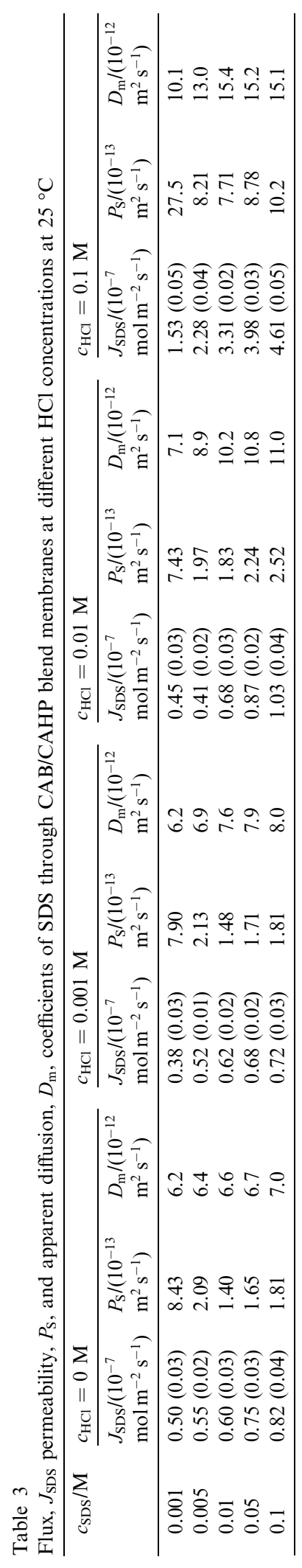

From the analysis of the $P_{\mathrm{S}}$ values we may remark: (a) at $c_{\text {SDS }}<\mathrm{cmc}$ the permeability coefficients decrease with an increase of SDS concentration; (b) at $c_{\mathrm{SDS}}>\mathrm{cmc}$ a slight increase in $P_{\mathrm{S}}$ is found. The first fact can be explained by the formation of hemimicelles which act as a further resistance to the whole SDS flux; the latter finding shows that although micelles do not permeate the membrane, they do produce an extra contributing factor to driving force of monomer diffusion. This occurs because it seems that at $c_{\mathrm{SDS}}>\mathrm{cmc}$ the HM formation reaches the saturation point. The presence of $\mathrm{HCl}$ only becomes significant to $P_{\mathrm{S}}$ at concentrations higher or equal to $10^{-2} \mathrm{M}$; at this concentration the effect on $P_{\mathrm{S}}$ is only found at concentrations above cmc, whilst at $0.1 \mathrm{M}$ $\mathrm{HCl}$ conditions a drastic increase of $P_{\mathrm{S}}$ occurs. These results also show that, although the effect of HM cannot be neglected, its effect decreases with the SDS association which is induced by the presence of $\mathrm{HCl}$.

The analysis of the apparent diffusion coefficients shows that in the absence of $\mathrm{HCl}$ almost no variation of $D_{\mathrm{m}}$ with concentration is observed, supporting the assumption that the monomer species are the only significant free diffusing species inside the membrane. As $\mathrm{HCl}$ concentration increases, the variation of $D_{\mathrm{m}}$, as a function of $c_{\mathrm{SDS}}$, increases too, in a significant way at $c_{\mathrm{SDS}}<\mathrm{cmc}$, showing that the resistance to the hydrodynamic flux decreases with an increase of free monomers; another possible explanation arises from a possible drag effect produced by the hydrogen ions. However at higher SDS concentrations, $D_{\mathrm{m}}$ values become approximately constant also showing that the micelles do not have a relevant role in the diffusion process.

\section{Conclusions}

The sorption of SDS on cellulose ester blend membranes occurs via hemimicelle formation in accordance with high distribution coefficients and increase in hydrophobicity of the surface as seen by fluorescence. Such an increase in the blend hydrophobicity leads to a consequent decrease in the water diffusion coefficient and in the permeability coefficient of the unimer. This occurs specially at concentrations where only unimer species exist; when micelles exist in solution, a small increase in the permeation is found, showing that the variation of this parameter depends on the $\mathrm{cmc}$. The analysis of the apparent diffusion coefficient leads to values approximately two orders of magnitude lower than in aqueous solution, showing that although the concentration of free diffusing unimers increases with concentration, the concentration tends to be very small. The spongy structure of cellulose as well as the presence of the polar groups of CAHP are suggested to be very important to mass transport by diffusion. It is known that the presence of hydrophilic groups, even in very small concen- 
tration, may control the diffusion process. This is suggested to be true also for the blend studied, even though the hydrophobic character of this is increased by formation of the SDS hemimicelles.

The hydrophilic/hydrophobic balance in the blend as well its effect on the transport properties of SDS can be modified by changing the $\mathrm{HCl}$ concentration. This may be used as an important tool to control the permeation of solutes through this kind of membranes. An increase of $P_{\mathrm{S}}$ and $D_{\mathrm{m}}$ is clearly found when $\mathrm{pH}$ increases, showing that SDS permeation can be controlled by altering the acidic concentration in the bulk solution, once the hydrogen ions will interact with the SDS, decreasing their association, and consequently changing the membrane properties.

Although the reported experimental results are not easy to interpret, we suggest a mechanism of transport and equilibrium involving the following steps: (i) the surfactant approaches the polymeric surface; (ii) hemimicelle formation occurs on the hydrophobic part of the blend, and, as a consequence, the polymeric membrane changes to have a more hydrophobic character; (iii) after a certain period of time all the ionic (hydrogen phthalate) and cellulose sites of the polymeric blend (both at the surface and most probably inside the polymer membrane) will be completely occupied, such that a "new" surface modified polymeric blend can be considered to exist, and to be accompanied by a relaxation of the membrane structure; (iv) in the presence of the acidic solute a synergetic mechanism may be involved: with the protonation of carboxylic groups, the $\mathrm{HCl}$ is available to decrease the SDS association and, as a consequence, the concentration of SDS which can dissolve inside the blend and diffuse will increase.

More experimental work is in progress to finally characterise this blend system. However, the present and previously reported experimental results show that such a matrix seems to have very promising features for use in separation processes since its dependence on hydrophobic/hydrophilic balance can be enhanced by the presence of SDS, and transport properties can be modulated by changing the acidity of the bulk solution.

\section{Acknowledgements}

We thank Professor Bjorn Lindman for valuable discussions. Financial support from POCTI/QUI/39593/ 2001 and POCTI/35415/QUI/2000 is gratefully acknowledged.

\section{References}

[1] Pelizetti E, Maurino V, Minero C, Pramauro E. In: Bloor DM, Wyn-Jones E, editors. The structure, dynamics and equilibrium properties of colloidal systems. Dordrecht: Kluwer; 1990. p. 325-53.

[2] Lu X, Winnik MA. Chem Mater 2001;13:3449.

[3] Roffey CG. Photopolymerization of surface coatings. Norwich: John Wiley \& Sons Ltd; 1985.

[4] Nyström B, Lindman B. Macromolecules 1995;28:967.

[5] Walderhaug H, Nyström B, Hansen F, Lindman B. J Phys Chem 1995;99:4672.

[6] Nyström B, Kjoniksen A-L, Lindman B. Langmuir 1996; 12:3233.

[7] Kjoniksen A-L, Nyström B, Lindman B. Macromolecules 1998;31:1852.

[8] Ostrovski D, Kjoniksen A-L, Nyström B, Torell LM. Macromolecules 1999;32:1534.

[9] Isarin J-C, Kaasjager ADJ, Holweg RBM. Textile Res J 1995;65:61.

[10] Upton BH, Krisnagopalan GH, Abubakr S. TAPPI J 1999;82:104.

[11] Valente AJM, Polishchuk AYa, Lobo VMM, Burrows H. Langmuir 2000;16:6475-9.

[12] Agar JN, Lobo VMM. J Chem Soc, Faraday Trans 1975; $71: 1659$.

[13] Lobo VMM, Valente AJM. Int J Polym Mater 1994;25:139.

[14] Lobo VMM, Valente AJM. Polym Degrad Stab 1994;44:147.

[15] Valente AJM, Burrows HD, Miguel MG, Lobo VMM. Eur Polym J 2002;38:2187.

[16] Polishchuk AYa, Valente AJM, Camino G, Luda MP, Madyuskin NN, Lobo VMM, et al. J Appl Polym Sci 2002;82:1157.

[17] Lobo VMM, Valente AJM, Polishchuk AYa, Geuskens G. J Mol Liquids 2001;94:179.

[18] Mills R. J Electroanal Chem 1965;9:57.

[19] Dong DC, Winnik MA. Can J Chem 1984;62:2560.

[20] Miguel MG. Adv Colloid Interface Sci 2001;89-90:1.

[21] Gaudin M, Fuerstenau DW. AIME Trans 1955;202:958.

[22] Chandar P, Somasundaran P, Turro NJ. J Colloid Interface Sci 1987;117:31.

[23] Chandar P, Somasundaran P, Waterman KC, Turro NJ. J Phys Chem 1987;91:148.

[24] Somasundaran P, Kunjappu JT, Kumar CV, Turro NJ, Barton JK. Langmuir 1989;5:215.

[25] Camino G, Polishchuk AYa, Luda MP, Revellino M, Blancon R, Martinez-Vega JJ. Polym Degrad Stab 1998; 61:53.

[26] Polishchuk AYa, Zaikov GE. Multicomponent transport in polymer systems for controlled release. Amsterdam: Gordon Breach Sci. Pub; 1997.

[27] Ng WK, Tam KC, Jenkins RD. J Rheol 2000;44:137.

[28] Childress AE, Deshmukh SS. Desalination 1998;118:167.

[29] Kameka N, Burgaud I, Zana R, Lindman B. J Phys Chem 1994;98:6785.

[30] Ghoreishi SM, Li Y, Bloor DM, Warr J, Wyn-Jones E. Langmuir 1999;15:4380.

[31] Nunes T, Burrows HD, Bastos M, Feio G, Gil MH. Polymer 1995;36:479.

[32] Stacké AM, Bock FC. Optimized cellulose membrane for artificial kidney dialysis application. Report No. IITRIU6059-9, April, 1970.

[33] Flockart BD. J Colloid Sci 1961;16:484.

[34] Kjøniksen A-L, Nilsson S, Thuresson K, Lindman B, Nyström B. Macromolecules 2000;33:877. 